\title{
Dendritic cells of the oral mucosa
}

\author{
A-H Hovav ${ }^{1}$
}

The oral cavity contains distinct mucosal surfaces, each with its own unique distribution of dendritic cell (DC) subsets. In addition to tissue-specific properties, such organization might confer differential immune outcomes guided by tissueresident DCs, which translate in the lymph node into an overall immune response. This process is further complicated by continual exposure and colonization of the oral cavity with enormous numbers of diverse microbes, some of which might induce destructive immunity. As a central cell type constantly monitoring changes in oral microbiota and orchestrating T-cell function, oral DCs are of major importance in deciding whether to induce immunity or tolerance. In this review, an overview of the phenotype and distribution of DCs in the oral mucosa is provided. In addition, the role of the various oral DC subsets in inducing immunity vs. tolerance, as well as their involvement in several oral pathologies is discussed.

\section{INTRODUCTION}

The term oral mucosa refers to the tissue that lines the oral cavity and is composed of the masticatory and lining mucosa. The masticatory mucosa forms the attached gingiva and hard palate, and its structure allows it to resist the abrasion of rough food particles. The lining mucosa represents the largest part of the oral mucosa, consisting of the buccal, sublingual, and vestibular mucosae. The two major layers of the oral mucosa, namely the epithelium and the underlying connective tissue (termed lamina propria), are equivalent to the epidermis and dermis of the skin. In most oral tissues, a submucosa layer can be found just beneath the lamina propria and is not easily discriminated from it. Similar to the epidermis, the oral epithelium maintains its structural integrity by a process of continuous cell renewal and migration from the deepest layers to the surface in order to replace those cells that were shed. The oral epithelium is a stratified (multi) layer of squamous cells that may be either keratinized or non-keratinized, with the latter being thicker and more permeable. Nonetheless, unlike the intestinal lining, the oral epithelium does not have an adsorptive capacity, although differences in permeability exist in direct correlation to the thickness of the epithelial barrier. ${ }^{1,2}$

The oral mucosa exploits various mechanisms to protect the host against invading pathogens. The tough but yet resilient lamina propria provides resistance to tear and compression forces, thus maintaining tissue integrity that is crucial in such an active site that is constantly exposed to microorganisms. Protection from microorganisms is also afforded by cell shedding from the surface layer, therefore minimizing colonization. Furthermore, salivary secretion aids in keeping the oral mucosa moist and limiting excessive bacterial accumulation. In addition to the aforementioned tissueintegral mechanisms, the oral mucosa contains an elaborate immune system. This system is thought to be pro-tolerogenic in nature as the oral mucosa remains in a relative state of health despite the heavy microbial load. ${ }^{3}$ Still, an inflammatory infiltrate composed mainly of lymphocytes and neutrophils can be regularly detected in the gingiva, even in those considered clinically healthy. ${ }^{4}$ This emphasizes the complicated mechanisms exerted by the oral immune system in order to maintain immunological homeostasis.

As the most potent cells activating and polarizing naive $\mathrm{T}$ cells, oral dendritic cells (DCs) are of major importance in induction of immunity and tolerance. DCs are a heterogeneous population of antigen-presenting cells (APCs) that are widely distributed in lymphoid and non-lymphoid tissues in the steady state. $^{5-7}$ These cells can be generally categorized into two classes: conventional DCs and plasmacytoid DCs (pDCs). In their immature state, conventional DCs constantly patrol tissue microenvironments, whereas upon encountering a foreign antigen they efficiently sample it via various pathways. The DCs then migrate to the draining lymph nodes (LNs) while undergoing a maturation process that enables the presentation of pathogen-derived peptides to $\mathrm{CD}^{+}{ }^{+}$and $\mathrm{CD} 8{ }^{+}$T cells. ${ }^{8}$ To induce accurate and efficient pathogen-specific immunity, DCs adjust their immune response to the type of pathogen. In

${ }^{1}$ Faculty of Dental Medicine, Institute of Dental Sciences, Hebrew University-Hadassah School of Dental Medicine, Jerusalem, Israel. Correspondence: A-H Hovav (avihaih@ekmd.huji.ac.il)

Received 16 August 2012; accepted 17 May 2013; published online 12 June 2013. doi:10.1038/mi.2013.42 
contrast to conventional DCs, pDCs are difficult to detect in peripheral tissues; they circulate in the blood and can be found in peripheral lymphoid organs. ${ }^{9,10}$ Upon infection, pDCs can enter inflamed tissues, produce type I interferon, and activate $\mathrm{T}$ cells in the LNs. ${ }^{11}$ In recent years, advanced immunological techniques and new mouse models have facilitated in vivo studies that have provided new insights into the developmental and functional aspects of DCs. This progress has also enriched our knowledge regarding the phenotype and function of oral DCs.

\section{PHENOTYPES IN THE MURINE ORAL MUCOSA}

Among the various distinct oral mucosal tissues, the buccal, gingival, and sublingual mucosa are the most studied with regard to their DC composition. This may be attributed to the accessibility of the tissue, similarity to a well-characterized nonoral tissue (buccal mucosa and skin), relevance to oral diseases (gingiva), and due to therapeutic interests (sublingual). Nevertheless, these tissues mirror adequately the DC heterogeneity of the oral mucosa, which is discussed below.

\section{BUCCAL MUCOSA}

Several mouse studies have demonstrated a high resemblance between the buccal mucosa and the skin immune system. At least four different DC subsets can be identified in the buccal tissue based on their expression of CD11c, CD103, Ep-CAM (epithelial cell adhesion molecule), and langerin (CD207): (1) Langerhans cells (LCs) located in the mucosal epithelium, (2) interstitial (or lamina propria) DCs (iDCs), which are the equivalent of dermal DCs, (3) langerin-expressing iDCs that also express the $\mathrm{CD} 103$ molecule $\left(\mathrm{Ln}^{+} \mathrm{iDCs}\right)$, and (4) langerin ${ }^{-} \mathrm{CD}_{103}{ }^{+} \mathrm{iDCs}^{12}$ (Figure 1). The latter DC population was identified in mice expressing the human diphtheria toxin receptor (DTR) under transcriptional control of the endogenous langerin/CD207 promoter (langerin-DTR). In these mice, a single administration of diphtheria toxin (DT) eliminates langerin-expressing cells (i.e., LCs and $\mathrm{Ln}^{+}$iDCs). Whereas $\mathrm{Ln}^{+}$iDCs return as soon as 3-5 days post-DT injection, repopulation of LCs occurs only after 2 weeks. This allows discrimination between these two DC subsets in vivo by employing a single vs. repeated injections of DT. The $\mathrm{CD} 103^{+}$ iDC subset was present in mice repeatedly administered with DT, suggesting that these cells do not express langerin like their counterpart $\mathrm{Ln}^{+}$iDCs. The presence of langerin-expressing iDCs in the buccal mucosa was also recently confirmed by another study. ${ }^{13}$ Of note, an additional population of APCs (presumably macrophages) expressing CD11b but not CD11c $\left(\mathrm{CD} 11 \mathrm{~b}^{+} \mathrm{CD} 11 \mathrm{c}^{-}\right)$exists in the buccal lamina propria in lower numbers than iDCs that express both markers.

\section{SUBLINGUAL MUCOSA}

The epithelium of the sublingual mucosa is particularly thin and thus provides a useful route of entry for antigens, allergens, and drugs. APCs in the sublingual mucosa have been analyzed by immunohistochemistry and flow cytometry. ${ }^{14,15}$ These techniques showed that the frequency of LCs in the sublingual epithelium is much lower than in that of the buccal mucosa. ${ }^{14}$ $\mathrm{CD}_{11 \mathrm{c}^{+}} \mathrm{CD} 1 \mathrm{~b}^{+}$cells (iDCs) and a large population of

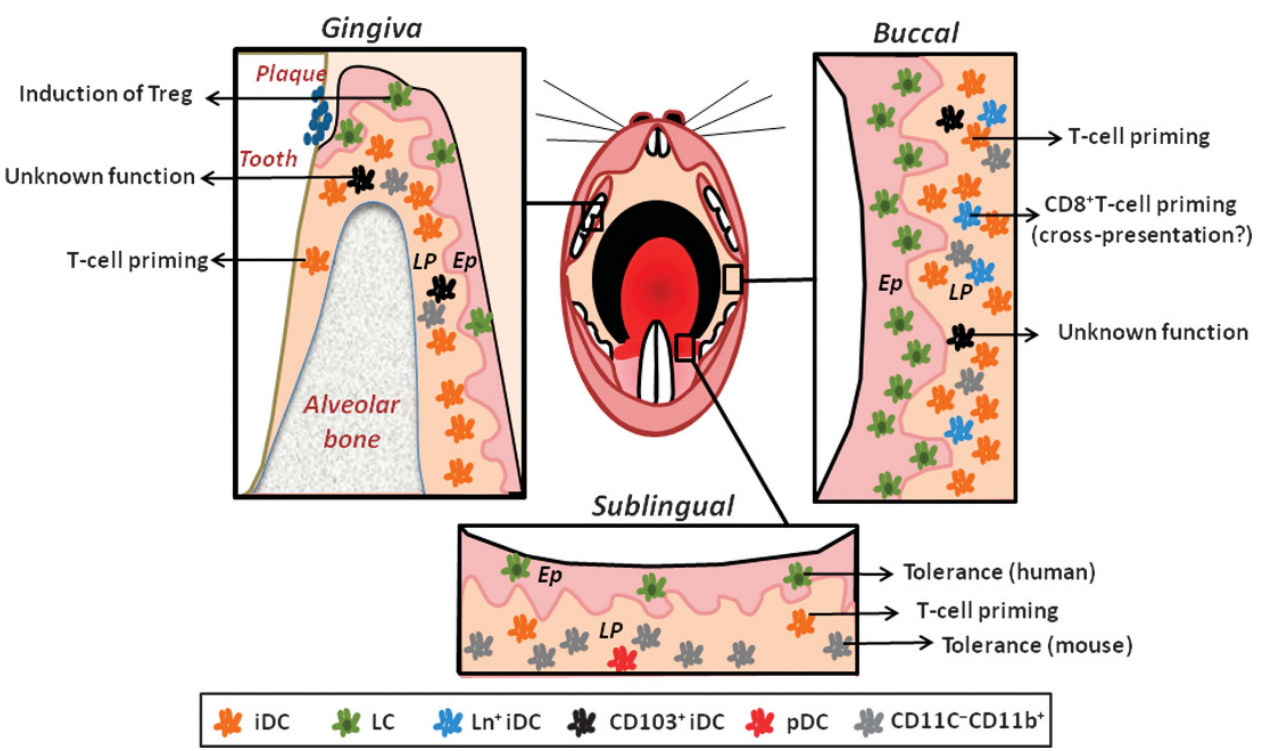

Figure 1 Schematic representation of the distribution and function of dendritic cells (DCs) in the buccal, gingival, and sublingual mucosae of the oral cavity. Langerhans cells (LCs) reside in the epithelium (Ep) of all three tissues, but the frequency of LCs in the buccal epithelium is considerably higher than those in the gingival and sublingual epithelia. The role of LC in the oral mucosa seems to be mainly tolerogenic. Interstitial DCs (iDCs) expressing both CD11c and CD11b represent the major DC subset in the lamina propria (LP) of the buccal and gingival mucosae; a small population of iDCs can also be found in the sublingual mucosa. iDCs appear to be the main oral DC subset mediating CD4 ${ }^{+}$and CD8 ${ }^{+} \mathrm{T}^{-c e l l ~ p r i m i n g}$ in the draining lymph nodes. $\mathrm{CD} 11 \mathrm{~b}^{+} \mathrm{CD} 11 \mathrm{c}^{-}$cells reside in all tissues but represent the majority of antigen-presenting cells in the sublingual tissue. These macrophage-like cells are shown to mediate tolerance against allergens in mice. The LP of the buccal and gingival mucosae contains a small fraction of $\mathrm{CD}_{103^{+}} \mathrm{iDC}$ s with unknown function; in the buccal mucosa, some of these cells also express langerin ( $\mathrm{Ln}^{+} \mathrm{iDCs}$ ) and might cross prime CD8 ${ }^{+} \mathrm{T}_{\mathrm{C}}$ cells. In a steady state, plasmacytoid DCs (pDCs) are found in the submucosal/muscular region of the sublingual mucosa. Under inflammatory conditions, pDCs infiltrate the oral mucosa. Treg, $T$ regulatory. 
$\mathrm{CD} 11 \mathrm{~b}^{+} \mathrm{CD} 11 \mathrm{c}^{-}$cells is located in the lamina propria/ submucosa interface. Interestingly, pDCs expressing $\mathrm{B} 220^{+} 120 \mathrm{Gr}^{+}$are also found, predominantly in the submucosa/muscular area. Another study reported that most APCs in this tissue $\left(\mathrm{CD} 11 \mathrm{~b}^{+} \mathrm{CD} 11 \mathrm{c}^{-}\right.$cells) are $\mathrm{F} 4 / 80^{+}$ macrophages, whereas most $\mathrm{CD}_{11 \mathrm{c}^{+}}$myeloid DCs also express CD11b (iDCs). ${ }^{15}$

\section{GINGIVAL MUCOSA}

The gingiva does not contain submucosa and the lamina propria is bound directly to the membrane that lines the outer surface of the alveolar bone. Low percentages of LCs $\left(\mathrm{CD} 11 \mathrm{c}^{+}\right.$ major histocompatibility complex (MHC) class $\mathrm{II}^{+}$langerin $^{+}$ and $\mathrm{Ep}-\mathrm{CAM}^{+}$) are located in the gingival epithelium in comparison to buccal and skin tissues. ${ }^{16}$ Most of the APCs express $\mathrm{CD} 11 \mathrm{c}^{+} \mathrm{CD}_{11} \mathrm{~b}^{+}$(iDCs), whereas $\mathrm{CD} 11 \mathrm{~b}^{+} \mathrm{CD} 11 \mathrm{c}^{-}$ cells are also present but in lower frequencies (A-H. Hovav, unpublished). A minor population of iDCs (about 5\%) also expresses the $\mathrm{CD} 103$ integrin. ${ }^{16} \mathrm{CD} 103{ }^{+} \mathrm{iDC}$ s that also express langerin are rarely found, thus doubting the presence of $\mathrm{Ln}^{+}$DCs in the gingiva (Table 1 ).

The phenotypes of DCs described above indicate that, despite constituting an entrance of the aerodigestive pathways, the oral mucosa contains distinct subsets of DCs in comparison to the lung and gut mucosa. ${ }^{17,18}$ This probably reflects histological and functional differences such as the type of the epithelium, a multi-layer in the mouth vs. a single layer in the gut and lung, resulting in the presence of LCs only in the oral mucosa. Still, the oral lamina propria contains $\mathrm{CD} 11 \mathrm{c}^{+}$ $\mathrm{CD}_{11 \mathrm{~b}^{+}}$DCs, which are also present in the lamina propria of the lung and small intestine. ${ }^{17,18}$ This is also true for $\mathrm{CD}_{103}{ }^{+}$DCs that are present in the lamina propria of the small intestine but seem to differ from those in the lung, which also express langerin and are located in the epithelial layer.

\section{PHENOTYPES IN THE HUMAN ORAL MUCOSA}

In contrast to mice, characterization of DCs in human oral tissues is limited, and it is generally focused on LCs. This could be related to the abundant presence of human oral LCs in the mucosa in comparison to other myeloid DCs and pDCs. ${ }^{19}$ Analysis of the gingiva identified langerin-expressing DCs (LCs) in the mucosal epithelium and CD209-positive DCs in the lamina propria, which are considered the equivalent of dermal DCs (Table 2). ${ }^{20}$ The majority of lamina propriaresident DCs also expressed the mannose receptor (CD206). Gingival LCs can be also identified in the epithelium based on the expression of the CD1a molecules. ${ }^{21}$ Nevertheless, although both langerin and CD1a can be used to classify LCs, langerin was reported to be a more specific marker. ${ }^{22}$ Three-dimensional analysis of gingival LCs demonstrated that these cells distribute their dendritic processes toward the gingival surface, reflecting their ability to monitor external stimuli. ${ }^{23}$ In a recent study, the distribution of LCs in various human oral tissues was examined based on the expression of CD1a. ${ }^{19}$ The highest numbers of LCs were found in the vestibulum, buccal, palate, and lingual tissues, while lower numbers were observed in the sublingual area and gingiva. Interestingly, these frequencies of LCs are similar to those observed in the mouse (buccal $>$ gingival $>$ sublingual), suggesting high resemblance in this regard between humans and mice. LCs from all human oral tissues

Table 1 DC subsets in murine oral mucosal tissues

\begin{tabular}{|c|c|c|c|c|c|c|c|c|c|c|c|}
\hline \multicolumn{12}{|c|}{ Cellular marker ${ }^{\mathrm{a}}$} \\
\hline Buccal & & & & & & & & & & & $12,13,28, U D$ \\
\hline LCs & + & + & + & + & + & $+/++$ & - & ND & ND & ND & \\
\hline $\mathrm{CD}_{103^{+}} \mathrm{DCs}$ & + & + & + & + & - & - & + & ND & ND & ND & \\
\hline $\mathrm{CD} 11 \mathrm{~b}^{+} \mathrm{CD} 11 \mathrm{c}^{-}$ & - & + & + & ++ & - & - & - & ND & ND & ND & \\
\hline Sublingual & & & & & & & & & & & 14,15, UD \\
\hline iDCs & + & + & + & ++ & - & - & - & ND & ND & - & \\
\hline LCs & + & + & + & + & + & + & - & ND & ND & - & \\
\hline $\mathrm{CD} 11 \mathrm{~b}^{+} \mathrm{CD} 11 \mathrm{c}^{-}$ & - & + & + & ++ & - & - & - & ND & ND & + & \\
\hline LCs & + & + & + & + & + & + & - & - & - & ND & \\
\hline $\mathrm{CD} 103^{+} \mathrm{DC}$ & + & + & + & ND & - & - & + & - & - & ND & \\
\hline$\underline{C D 11 b}{ }^{+} \mathrm{CD}_{11 c^{-}}$ & - & + & + & ++ & - & - & - & - & - & ND & \\
\hline
\end{tabular}


Table 2 DC subsets in human oral mucosal tissues

\begin{tabular}{|c|c|c|c|}
\hline $\begin{array}{l}\text { DC } \\
\text { subsets }\end{array}$ & Cellular markers ${ }^{a}$ & & References \\
\hline LCs & $\begin{array}{l}\text { Langerin, CD1a, MHC class II, CD14, mannose } \\
\text { receptor(CD206), DEC-205(CD205), FCERI, FcyR, } \\
\text { TLR2, TLR4, CD11b }\end{array}$ & $\begin{array}{l}\text { Frequency: buccal > gingival > sublingual } \\
\text { Express CD83, DC-LAMP(CD208) and CCR7 upon maturation } \\
\text { Tolerance induction }\end{array}$ & $\begin{array}{l}19,20,24,42 \\
49-51,59,79 \\
126\end{array}$ \\
\hline mDCs & $\begin{array}{l}\text { DC-SIGN(CD209), MHC class II, CD11C, mannose } \\
\text { receptor(CD206) }\end{array}$ & $\begin{array}{l}\text { Immunity } \\
\text { Express CD83, DC-LAMP(CD208) and CCR7 upon maturation } \\
\text { Possible involvement in Sjögren's syndrome }\end{array}$ & $20,24,59,127$ \\
\hline
\end{tabular}

Abbreviations: CCR, C-C motif chemokine receptor; DC-LAMP, dendritic cell lysosomal-associated membrane protein; DC-SIGN, dendritic cell-specific intercellular adhesion molecule-3-grabbing non-integrin; FcyR, IgG Fc receptors; FCeRl, high-affinity receptor for the Fc region of immunoglobulin E (IgE); LCs, Langerhans cells; mDCs, myeloid dendritic cells; MHC, major histocompatibility complex; pDCs, plasmacytoid DCs; TLR, Toll-like receptor.

${ }^{a}$ Cellular markers thought to be specific for the noted DC subset are in bold.

expressed also langerin, albeit in a lower intensity than epidermal LCs. Furthermore, these LCs express similar levels of MHC class II and the CD83 maturation marker, suggesting that oral LCs from all tissues are in a similar maturation stage in steady state. ${ }^{19}$ With regard to pDCs, these cells are present in low frequencies in healthy oral tissues, whereas under inflammatory conditions, significant infiltration of $\mathrm{CD} 123^{+}$ BDCA-2 ${ }^{+}$pDCs to the epithelium and lamina propria can be seen. $^{24}$

\section{ANTIGEN CAPTURE AND MIGRATIONAL CAPABILITIES OF ORAL MUCOSAL DCs}

Because of the relatively non-adsorptive nature of the oral mucosa, it is thought that the antigen has to activate or penetrate the epithelium in order to elicit potent immune responses. This could be achieved by mechanical means (i.e., needle, microneedle, and mucoadhesive nanoparticles) or by the intrinsic invasion capability of the immunological insult. The sublingual mucosa is an exception, as the antigen can be easily adsorbed and reach DCs. ${ }^{25}$ Once capturing antigen, migration of DCs into the draining LNs is critical for the induction of protective immunity as well as for immunological tolerance. ${ }^{26,27}$ Following exposure to a pathogen, DCs mature en route to the $\mathrm{LNs}$ where they encounter $\mathrm{T}$ cells and present the antigen. Although the murine oral mucosa drains directly to the cervical LNs, in humans, distinct LNs drain different areas of the oral cavity that then reach the superficial and deep cervical LNs. Several studies have examined the ability of DCs from various oral mucosal tissues to travel to the draining LNs. In concurrence with the similarity between buccal and skin DCs, migration kinetics of buccal mucosa DCs to the $\mathrm{LN}$ is quite comparable with that of skin DCs, albeit in lower numbers. ${ }^{28}$ By applying fluorescein isothiocyanate (FITC) and the hapten dibutyl phthalate (DBP) to the mouse buccal tissue, it was shown that whereas iDCs rapidly migrate to the LNs, LCs are much slower, peaking at $96 \mathrm{~h}$ after hapten application. A more thorough examination demonstrated that buccal LCs migrated to the LNs at a slower rate than epidermal LCs. ${ }^{13}$ Moreover, in this experimental setting, buccal LCs were found to express lower levels of the co-stimulatory molecules CD40, CD86, CD273 (B7-DC/PD-L2), and CD274 (B7-H1/PD-L1) as compared with other buccal-derived DCs. Aside from these two subsets, a third population of DCs expressing $\mathrm{CD} 11 \mathrm{c}^{\text {intermeidate/low }}$ also migrated from the buccal mucosa, representing a newly recruited DC subset arriving after FITC application. These cells migrated continuously to the LNs and maintained the highest ratio of all the three subsets. ${ }^{28}$ Rapid recruitment (within 2-6h) of inflammatory cells to the buccal mucosa was also observed following topical delivery of DNFB (1-fluoro-2,4-dinitrobenzene) hapten or immunization with measles virus nucleoprotein. ${ }^{29-31}$ This recruitment was mediated by the chemokine receptor 6 (C-C motif chemokine receptor 6 (CCR6))/chemokine ligand 20 (C-C motif chemokine ligand 20 (CCL20)) pathway and could be enhanced by FMS-like tyrosine kinase 3 treatment. $^{30,31}$ Similar migration of DCs from the buccal and skin tissues was reported after immunization with plasmid DNA. ${ }^{12,32}$ Although not verified, it is likely that buccal DCs employ the CCR7-CCL19/CCL21 axis to migrate to the LN, like skin DCs. ${ }^{33,34}$ Expression of CCR7 in activated buccal mucosa is in line with this assumption. ${ }^{35}$ The CCR7-CCL19/CCL21 axis has also been shown to mediate DC migration from the sublingual mucosa. ${ }^{15}$ Following sublingual application of an antigen, antigen-bearing DCs can be detected in the cervical LNs as soon as $6 \mathrm{~h}$ later, with maximal trafficking observed $12 \mathrm{~h}$ after the application. ${ }^{36}$ Finally, migration of gingival DCs to the draining cervical LNs was observed following application of FITC/DBP solution or infection with the oral pathogen Porphyromonas gingivalis. ${ }^{16}$ Although the precise migration kinetics of each DC subset was not reported, antigen-experienced iDCs, LCs, and $\mathrm{CD}_{103}{ }^{+} \mathrm{iDCs}$ were found in the LNs 3 days post exposure to P. gingivalis.

\section{ANTIGEN PRESENTATION AND T-CELL INDUCTION BY ORAL DCs}

Despite being considered a tolerogenic environment, $T$ cells can be efficiently induced by DCs in the oral mucosal immune system. Numerous studies have shown the induction of antigen-specific T-cell responses by buccal DCs following 
topical or penetrable administration of hapten, protein antigen, and plasmid DNA. ${ }^{12,29,30,37,38}$ The sublingual mucosa has also been shown to initiate broad systemic and mucosal antigenspecific protective immune responses (reviewed by Czerkinsky and Holmgren ${ }^{39}$ ). Moreover, immune responses induced by sublingual immunization have been reported to surpass those achieved by intramuscular, traditional intranasal, or other oral mucosal immunizations. ${ }^{40}$ Sublingual administration of adenoviral vectors was able to prime potent protective pathogen-specific $\mathrm{T}$ cells and even overcome pre-existing immunity. ${ }^{40,41}$ Taken together, these studies provide compelling evidence for T-cell activation by oral DCs upon migration to the LNs.

Nevertheless, migration of tissue-derived DCs to the LNs following infection/immunization does not always indicate that these migratory DCs will directly activate $\mathrm{T}$ cells. For example, skin-derived DCs efficiently prime $\mathrm{T}$ cells following immunization with lentivirus but fail to do so after infection with herpes simplex virus type 1 (HSV-1). ${ }^{42-44}$ The reason for this discrepancy is that HSV-1, but not lentivirus, interferes with the capability of infected DCs to present antigen. ${ }^{45}$ With regard to HSV-1, it was also suggested that the route of delivery greatly impacts the type of DCs presenting viral antigens. Whereas mucosal infection with HSV-1 triggered direct presentation by mucosal-resident DCs, administration of the virus by needle injection resulted in presentation by $\mathrm{LN}$-resident DCs. ${ }^{46}$ These findings suggest that the type of immunological insult, as well as the route of entry, have a major impact on the antigenpresenting abilities of oral DCs.

In a recent study examining the mechanisms of immune induction by buccal DCs following DNA vaccination, delayed antigen-presenting activity was observed in mice. ${ }^{12}$ Presentation of the antigen to $\mathrm{CD} 8^{+} \mathrm{T}$ cells was mediated mainly by buccal-derived iDCs, while $\mathrm{Ln}^{+}$iDCs showed limited presentation. This activity is similar to the antigen-presenting activity observed following intradermal immunization with plasmid DNA. ${ }^{32}$ The skin and buccal tissues also presented similar kinetics of antigen expression, exhibiting maximal expression of the plasmid-encoded antigen $24-48 \mathrm{~h}$ post immunization. However, despite the high similarity among these tissues, major differences were found as well. First, restricting antigen expression to $\mathrm{CD}_{11 \mathrm{c}^{+}}$cells (i.e., DCs) abrogated $\mathrm{CD} 8{ }^{+} \mathrm{T}$-cell responses in the buccal tissues but not in the skin. This suggests that following plasmid DNA immunization, buccal DCs rely on cross-presentation to prime $\mathrm{CD}^{+} \mathrm{T}$ cells. Cross-presentation refers to the ability of certain DC subsets to transfer exogenously derived antigen to the MHC class I, instead of MHC class II pathway, in order to present it to $\mathrm{CD} 8{ }^{+} \mathrm{T}$ cells. ${ }^{47}$ It can thus be proposed that some buccal DCs have cross-presenting capabilities. Second, in the absence of LCs, CD8 ${ }^{+}$T-cell responses induced by buccal immunization with plasmid DNA were considerably reduced, whereas no effect was observed in intradermal immunization. ${ }^{12,32}$ Collectively, these observations suggest that DCs of the buccal mucosa engage unique mechanisms for immune induction; therefore, care should be taken when comparing DC function between skin and buccal tissues.
Antigen presentation was also examined following sublingual vaccination using the ovalbumin antigen and cholera toxin as an adjuvant. ${ }^{15}$ In this system, robust proliferation of $\mathrm{CD}^{+}{ }^{+} \mathrm{T}$ cells, and to a lesser extent $\mathrm{CD} 8{ }^{+} \mathrm{T}$ cells, was observed primarily in the cervical LNs. Sublingual iDCs were shown to transfer the antigen to the LNs, and presentation of the antigen to $\mathrm{CD} 4^{+} \mathrm{T}$ cells was mediated by both sublingual migratory DCs and LN-resident DCs. Ablation of langerin-expressing cells (i.e., LCs) in this model had no effect on antigen presentation. Interestingly, in a recent study sublingual iDCs were shown to be dispensable in eliciting tolerance against ovalbumin, a function that was mediated by oral macrophagelike $\left(\mathrm{CD} 11 \mathrm{~b}^{+} \mathrm{CD} 11 \mathrm{c}^{-}\right)$cells. ${ }^{36}$ It is thus likely that in a mouse model sublingual iDCs primarily induce immunity, whereas $\mathrm{CD} 11 \mathrm{~b}^{+} \mathrm{CD} 11 \mathrm{c}^{-}$cells are involved in mediating tolerance.

The ability of gingival DCs to present antigen and prime $\mathrm{T}$ cells was examined almost solely in the setting of experimental periodontal diseases. In this model, mice are infected several times with the oral pathogen $P$. gingivalis by oral gavage, resulting in local inflammation and $\mathrm{CD} 4{ }^{+}$T-cell-mediated bone loss. ${ }^{16} P$. gingivalis-derived peptides were presented mainly by iDCs to both $\mathrm{CD}^{+}$and $\mathrm{CD}^{+} \mathrm{T}$ cells. These findings suggest that iDCs located in the lamina propria can take up oral pathogen administered by gavage. This may be explained by the invasiveness of $P$. gingivalis. ${ }^{48}$ LCs were not able to present antigen and activate $\mathrm{T}$ cells. However, in their absence, activation of $\mathrm{CD} 4^{+} \mathrm{T}$ cells was increased, suggesting a regulatory role for LCs in experimental periodontitis. ${ }^{16} \mathrm{LN}$ resident DCs also presented antigens to $\mathrm{T}$ cells, albeit at a lower magnitude. As live bacteria were not found in the draining LNs, it is likely that LN-resident DCs acquired antigens arriving via the lymph or from migrating oral DCs. $\mathrm{CD} 103^{+}$iDCs were able to present antigen only to $\mathrm{CD} 8{ }^{+} \mathrm{T}$ cells, albeit to some extent.

\section{INDUCTION OF TOLERANCE BY ORAL DCs}

Despite continuous exposure to large amounts of antigens derived from food and microbes, the latter could be either commensal or pathogenic, severe inflammatory responses in the oral cavity are relatively rare. ${ }^{3}$ This is attributed to the tolerogenic properties of the oral mucosa that are critical for maintaining immunological homeostasis. Whereas the mechanisms involved in this process are a subject of extensive investigation, the oral cavity is an attractive site for inducing tolerance against allergens. Particular attention was given to the sublingual mucosa as a site of allergen application.

Studies employing human oral LCs demonstrated higher expression of Toll-like receptors 2 and 4 (TLR2 and TLR4) on oral LCs in comparison to epidermal LCs in steady state. ${ }^{49}$ In a response to TLR triggering with lipopolysaccharide, oral LCs upregulate expression of the co-inhibitory molecules B7-H1 and $\mathrm{B} 7-\mathrm{H} 3$, whereas expression of the co-stimulatory molecule CD86 (B7-2) is decreased. This resulted in a polarized development of $\mathrm{T}$ regulatory (Treg) cells and secretion of interleukin (IL)-10 and transforming growth factor- $\beta$ by stimulated T cells. ${ }^{50}$ This suggests that human oral LCs, which are located in the mucosal epithelium and thus exposed 
constantly to microbial stimuli, act in a tolerogenic fashion to maintain immune homeostasis. An additional support for the repeated exposure of human oral LCs to microbes could be reflected by their higher activation state in the tissue as compared with epidermal LCs. Oral LCs express higher levels of MHC class II, CD86(B7-2), CD80(B7-1), and CD40 molecules and are more effective in stimulating allogeneic $\mathrm{T}$ cells than epidermal LCs. ${ }^{51-53}$ Of note, expression of the CD83 maturation marker was similar in oral and epidermal LCs, suggesting a state of partial maturation in oral DCs, which may facilitate tolerance as previously proposed. ${ }^{7,54}$ Interestingly, partial maturation of oral LCs with tolerogenic properties was observed recently in an ex vivo model of allergen application, a phenomenon that was not detected in epidermal DCs under similar conditions. ${ }^{49}$

In contrast to the important role of human sublingual LCs in tolerance induction, mouse studies suggested that these cells are dispensable in this process. Instead, macrophage-like F4/80 $\mathrm{CD}_{11 \mathrm{~b}}{ }^{+} \mathrm{CD} 11 \mathrm{c}^{-}$cells were shown to polarize T cells into Treg expressing both interferon (IFN)- $\gamma$ and IL-10 cytokines. ${ }^{36}$ Furthermore, similar to the tolerogenic macrophages found in the lamina propria of the small intestine, the sublingual $\mathrm{CD}_{11 \mathrm{~b}}{ }^{+} \mathrm{CD}_{11 \mathrm{c}^{-}}$cells express mannose receptor (CD206) and class- 2 retinaldehyde dehydrogenase. This dissimilarity could be explained by the relatively lower numbers of LCs in the sublingual mucosa of mice in comparison to human. ${ }^{14,50}$ Nonetheless, studies in both human and mice point out that the sublingual mucosa is an attractive immunological site to induce tolerance.

\section{BALANCING BETWEEN TOLERANCE AND IMMUNITY}

The ability of oral DCs to mediate either immunity or tolerance highlights the question of how these cells discriminate between commensals and pathogens. Similar to oral pathogens, oral commensals can induce maturation and activation of monocyte-derived human DCs. ${ }^{55}$ Thus, it is likely that additional mechanisms other than just a direct recognition of pathogenspecific molecules might be involved. It has been proposed that in the intestine, tissue-resident DCs are educated by epithelial cells to be tolerogenic, whereas newly infiltrating DCs induce inflammatory responses. ${ }^{56}$ In such a scenario, DCs can tolerate commensals, whereas pathogens capable of invading or activating epithelial cells will trigger the recruitment of proinflammatory DCs that will elicit immunity. This could also be the case in the oral cavity, and it might also explain the enhanced tolerogenic properties of the sublingual tissue, which contains a thin epithelium that might be more vulnerable for invasion. Still, in contrast to the intestine, the oral epithelium is a multi-layer tissue that harbors LCs, and thus pathogen recognition might involve additional mechanisms. One possibility is the ability of microbes to cross the epithelium and reach the lamina propria, where iDCs can sample it and activate T cells. ${ }^{57}$ We recently showed that iDCs prime $P$. gingivalisspecific $\mathrm{T}$ cells following oral gavage, suggesting that $P$. gingivalis might traverse the gingival epithelium, as previously suggested. ${ }^{16,57}$ A recent study proposed that oral pathogen can alter the balance of the oral commensals, which subsequently facilitate local destructive immune responses. ${ }^{58}$ It is thus possible that under certain conditions commensals can also act as pathogens and activate the immune system. Such a situation might happen when immune regulatory cells such as oral LCs are impaired by constant activation or due to aging. Collectively, it is likely that the decision to react against a certain microorganism depends on its potential to invade the oral mucosa or alter its microbial homeostasis, as well as by the integrity of the oral immune system.

\section{DO ORAL LYMPHOID FOCI EXIST?}

In contrast to other mucosal tissues, which generally contain mucosa-associated lymphoid tissue (MALT) for local immune induction, the oral mucosa lacks such immunological structure. The MALT resembles a lymphoid organ as it contains T cells, B cells, plasma cells, and APCs such as DCs and macrophages, which are well situated to encounter antigens passing through the mucosal epithelium. In the case of Payer's patches of the intestine, the antigen is sampled by specialized $M$ cells that transfer it from the lumen to the lymphoid tissue.

Studies analyzing human gingiva during periodontitis have noticed an accumulation of mature $\mathrm{CD} 83^{+}$DCs in the T-cellrich area of the lamina propria. ${ }^{59}$ In that study, the authors also assumed that LCs migrating from the epithelium contributed to the pool of mature DCs in the lamina propria that interact with T cells. Similar co-localization of DCs and lymphocytes in the lamina propria was also observed by another study. ${ }^{60}$ Based on these findings, the concept of oral lymphoid foci was established, suggesting that inflamed gingiva might serve as a site for immune induction rather than just an effector site during periodontitis. Interestingly, the notion of oral lymphoid foci was also recently proposed in the context of tolerance induction. It was shown that besides exhibiting a partial maturation state, allergen-experienced LCs migrating from oral tissue explant expressed lower CCR7 levels compared with other migratory LCs. ${ }^{49}$ As CCR7 mediate migration to the LNs, the authors speculated that low expression of this chemokine receptor might imply migration of LCs to the lamina propria rather than to the draining LNs. Nevertheless, in both cases, there are no direct evidences for the migration and accumulation of mature LCs in the lamina propria. It was demonstrated that LCs alter the expression of matrix metalloproteinases 2 and 9 and their tissue inhibitors during periodontitis, suggesting migration of these cells towards the connective tissue, thus supporting their accumulation in the lamina propria. ${ }^{61}$ Still, such movement might represent migration to lymphatic vessels that directs LCs to the draining LNs. Another caveat in the concept of oral lymphoid foci is the absence of B cells. ${ }^{59}$ As abovementioned, in addition to T cells and APCs, the MALT contains also B cells. It is possible that B cells were overlooked during the immunohistochemical analysis of the inflamed gingiva, as another study employing flow cytometry analysis demonstrated that the majority of $\mathrm{CD} 83^{+}$cells in the diseased gingiva were, in fact, B cells. ${ }^{62}$ Indeed, the presence of CD $83^{+} \mathrm{B}$ cells in the lamina propria was confirmed shortly also by 
immunohistochemistry, although the relative contribution of DCs and B cells to this mature APC pool is not clear. ${ }^{20}$ In this regard, B cells were reported to promote expansion of Treg cells in tolerance induced by allergen application via the oral mucosa. ${ }^{63}$ Also, IL-10-producing B cells were found to restrain inflammation by promoting differentiation of immunoregulatory over pro-inflammatory T cells. ${ }^{64}$ It is thus possible that the oral lymphoid foci are, in fact, sites in which antigenexperienced mature B cells interact with $\mathrm{T}$ cells. Such interaction could facilitate the generation of Treg cells either for tolerance induction or in order to control inflammation during periodontitis. Further investigation is required to verify whether lymphoid foci exist in oral mucosa and what their roles are in immune induction.

\section{THE IMPACT OF AGING AND SMOKING ON ORAL DCs}

Accumulating data from non-oral tissues suggested that DC's function is hampered with age. Although there is no considerable impact on the number and phenotype of myeloid DCs in the blood of healthy aged human, ${ }^{65-67}$ their capability to sample antigen, migrate to the LNs, and prime $\mathrm{T}$ cells is diminished. ${ }^{65,68-70}$ Aging appears to compromise the ability of DCs to accurately direct immunity vs. tolerance. This could be attributed, in part, to the basic tendency of aged DCs to produce pro-inflammatory cytokines, ${ }^{71}$ which decreases their stimulation upon exposure to foreign antigen while increasing response against self antigens. ${ }^{71-75}$ Interestingly, in contrast to the above observations in the blood, the density of LCs in healthy gingival epithelium was shown to be reduced in aged subjects as compared with young. ${ }^{76}$ Such age-related differences in the density of LCs were not observed in other healthy human oral tissues, ${ }^{77}$ while existing in murine oral samples. ${ }^{78}$ With regard to their morphology, LCs showed fewer dendriticbranching processes and had a rounded shape in the older age group. Reduced numbers of LCs in the gingival epithelium of older individuals was also found during chronic periodontitis. $^{79,80}$ In the latter case, LCs of older subjects were located in the upper epithelium and their dendritic processes were shorter and less numerous. The obvious differences between the gingiva and other oral tissues might be related to the presence of a chronic low-grade inflammatory state in the gingiva. ${ }^{81-83}$ This might lead to gradual decline in the integrity of the local immune cells, particularly LCs being crucial for maintaining tolerance. ${ }^{16,84,85}$ Of note, an age-dependent decrease in the number of LCs was also observed in the epidermis. ${ }^{68,86}$ Similar to the gingival epithelium, this might be partly due to the result of constant exposure to sunlight, which was shown to reduce epidermal LCs numbers. ${ }^{87}$

Smoking is a significant risk factor for various oral pathologies. ${ }^{88}$ Although the precise mechanisms remain to be elucidated, there are observations that tobacco smoking can affect oral DCs. Nicotine, a constituent of cigarette smoke, was recently shown to modulate the immunoregulatory function of DCs that were exposed to lipopolysaccharide of $P$. gingivalis. ${ }^{89}$ Nicotine-experienced DCs preferentially induced Thelper type 2 (Th2) responses and decreased Th1 responses, an activity that was partially mediated by upregulation of the peroxisome proliferator-activated receptors- $\gamma$, which has immunomodulatory properties. ${ }^{90}$ In agreement with the ability of smoking to modulate DC function in vitro, it was shown that smoking reduces the ability to mount and maintain an effective defense reaction to plaque accumulation. ${ }^{91}$ This could be attributed to a decrease in the quantitative distribution of oral LCs and DCs during chronic gingivitis in smokers. ${ }^{92}$ A decrease in the density of oral LCs was observed in the context of smokeless tobacco as well. ${ }^{93}$ Taken together, it is likely that smoking alters oral DC function in a manner that might facilitate the development of oral diseases.

\section{THE INVOLVEMENT OF DCS IN ORAL PATHOLOGIES}

As DCs are essential for initiating immune responses, they also have a central role in the generation and perpetuation of immune-mediated diseases. Indeed, DCs of the oral mucosa were demonstrated to be directly involved in various oral diseases. Furthermore, a very recent study even proposed the involvement of DCs harboring oral pathogens during chronic periodontitis with coronary artery disease. ${ }^{94}$ This suggests that besides of being involved in oral diseases, oral DCs might also have a systemic impact on the host.

\section{PERIODONTITIS}

It is well established that whereas bacterial plaque is essential for the initiation and progression of chronic periodontitis, tissue damage is mediated primarily by the host immune response developed against the accumulating bacteria. ${ }^{95}$ In a murine model of periodontitis, $\mathrm{CD} 4{ }^{+} \mathrm{T}$ cells were found to have a critical role in the destruction of alveolar bone, the hallmark of this disease. ${ }^{96}$ Particularly, expression of receptor activator of NF- $\kappa B$ ligand (RANKL), a molecule involved in the regulation of bone remodeling by $\mathrm{CD} 4^{+} \mathrm{T}$ cells, was proposed to have a critical influence on bone resorption. ${ }^{97,98}$ As the key cells activating and regulating T cells, DCs and LCs were suggested to have an important role in the transition of human periodontitis-associated immune responses from protective to destructive. LCs seems to be of major importance in this aspect due to their localization in the gingival sulcular epithelium, thus putting them in a perfect position to survey the development of bacterial plaque. ${ }^{19,23,99}$ In agreement, the number of LCs in the gingiva increases as dental plaque accumulates, whereas its removal results in their decrease. $^{100-102}$ These observations suggest that oral LCs respond to changes in the oral microbiota and local inflammatory status. Further verifications to this hypothesis were obtained from analysis of individuals with chronic periodontitis in which lower numbers of LCs were found in the oral epithelium as compared with gingivitis or health. ${ }^{103,104}$ Moreover, it was demonstrated that the numbers of LCs are significantly reduced as periodontal disease progressed. ${ }^{105,106}$ It should also be noted that aging and smoking increase the prevalence of periodontitis, ${ }^{107,108}$ two conditions by which epithelial LCs network is damaged. ${ }^{76,77,92}$ Based on these observations, a recent study was set up to 
directly address the role of LCs in experimental periodontitis. ${ }^{16}$ Using Langerin-DTR mice to ablate LCs in vivo, it was shown that the absence of LCs during experimental periodontitis increased alveolar bone loss. LCs were shown to downregulate the generation of destructive RANKL-expressing $\mathrm{CD} 4^{+} \mathrm{T}$ cells by inducing higher numbers of Treg cells. These results proposed a protective role for oral LCs during periodontitis.

\section{ORAL LICHEN PLANUS}

Oral lichen planus (OLP) is a chronic oral inflammatory disease of unknown etiology. The disease is characterized by a massive infiltration of lymphocytes, mainly $\mathrm{T}$ cells, which chronically destructs the epithelium basal layer resulting in an abnormal epithelial keratinization cycle. Early studies demonstrated the presence of activated LCs/DCs in the OLP infiltrate, suggesting their involvement in disease progression. ${ }^{109,110}$ Although discrepancies exist regarding the numbers of LCs in OLP tissues ${ }^{24110-113}$ (probably due to the differences in tissue sampling and techniques), it suggests that LCs might have a role in the disease. More recent studies identified accumulation of mature LCs and DCs in the submucosa of OLP biopsies, and these cells were co-localized with lymphocytes. ${ }^{111,113}$ Such cellular organization was proposed to allow ongoing presentation of self antigen to $\mathrm{T}$ cells that facilitate tissue destruction. Interestingly, such contact between mature DCs and T cells in the oral mucosa, which was observed also by other studies, ${ }^{114,115}$ supports the concept of the oral lymphoid foci discussed above. Beside myeloid DCs, pDCs were also observed in OLP and were found adjacent to mature DCs. ${ }^{24}$ These pDCs were shown to secrete IFN- $\alpha$ and to contain granzyme $B$, thus proposed to facilitate DC maturation and to synergize lymphocytes in tissue destruction.

\section{SJÖGREN'S SYNDROME}

Sjögren's syndrome is a chronic systemic inflammatory and lymphoproliferative autoimmune disease of unknown etiology. It is characterized by dryness of the mouth and eyes due to progressive infiltration of immune cells into the salivary and lacrimal glands, which decreases their glandular secretion. $\mathrm{CD} 4{ }^{+} \mathrm{T}$ cells with cytotoxic capability were shown to be the predominant cells mediating infiltration into the glands; still, $\mathrm{CD}^{+} \mathrm{T}$ cells, B cells, and plasma cells are also present. ${ }^{116,117}$ As activation of the type I IFN system has been described in the salivary gland of Sjögren's syndrome patients, pDCs were proposed to have a role in the disease. ${ }^{118,119}$ pDCs are considered to be the main type I IFN-producing cells, ${ }^{120,121}$ and analysis of diseased salivary glands identified IFN- $\alpha$ producing cells while no pDCs were observed in healthy glands. Interestingly, the increase in pDC numbers in the salivary gland was accompanied by a reduction of their numbers in the blood. ${ }^{122}$ In addition to pDCs, myeloid DCs also seem to have a role in the pathogenesis of Sjögren's syndrome. It was shown that the levels of blood myeloid DCs decrease in the peripheral blood of Sjögren's syndrome patients, while preferential trafficking of these cells to salivary glands was observed in the early stage of the disease. ${ }^{123,124}$ Following entry to the glands, myeloid DCs were proposed to activate Th1 cells. ${ }^{123} \mathrm{~A}$ contribution of infiltrating monocytes to the pool of mature DCs in the salivary gland was also suggested. ${ }^{125}$

\section{CONCLUDING REMARKS}

The complexity of the oral immune system might reflect the diverse immunological challenges constantly faced by this tissue. DCs have a critical role in linking innate and adaptive immunity, as well as in mediating immunity or tolerance. Thus, oral DCs should be thoroughly studied in our attempt to understand oral immunity. Unfortunately, we know very little about the function of oral DCs, and as indicated in this review, it is not always possible to extrapolate their function from their counterparts in non-oral tissues. Future works should investigate how DCs in different oral immunological niches respond to a given immunological insult, and how simultaneous activation of these sites is integrated in the LNs for an overall immune response. In addition, it is important to characterize the type of monocytes entering the oral mucosa during inflammation. Some of these infiltrating cells could differentiate into DCs and may be involved in immune induction. Answering such questions will increase our knowledge of the mechanisms engaged by the oral immune system, facilitating their translation into novel therapeutic approaches.

\section{ACKNOWLEDGEMENTS}

This work was supported by the Israel Science Foundation (grant no. 1418/ 11 to $\mathrm{A}-\mathrm{HH}$ ), the German Israeli Foundation for young investigators (GIF Young), and the Dr. I. Cabakoff Research Endowment Fund at the Hebrew University-Hadassah School of Dental Medicine, Jerusalem, Israel.

\section{DISCLOSURE}

The author declared no conflict of interest.

c 2014 Society for Mucosal Immunology

\section{REFERENCES}

1. Lavelle, C.L.B. Applied Oral Physiology, 2nd edn, pp 244 (Wright Publishing Company, Costa Mesa, CA, USA, 1988).

2. Nanci, A. Ten Cate's Oral Histology: Development, Structure and Function, 7th edn, pp 411 (Mosby Elsevier, St. Louis, MO, USA, 2008).

3. Novak, N., Haberstok, J., Bieber, T. \& Allam, J.P. The immune privilege of the oral mucosa. Trends Mol. Med. 14, 191-198 (2008).

4. Page, R.C. \& Schroeder, H.E. Pathogenesis of inflammatory periodontal disease. A summary of current work. Lab. Invest. 34, 235-249 (1976).

5. Banchereau, J. \& Steinman, R.M. Dendritic cells and the control of immunity. Nature 392, 245-252 (1998).

6. Helft, J., Ginhoux, F., Bogunovic, M. \& Merad, M. Origin and functional heterogeneity of non-lymphoid tissue dendritic cells in mice. Immunol. Rev. 234, 55-75 (2010).

7. Steinman, R.M., Hawiger, D. \& Nussenzweig, M.C. Tolerogenic dendritic cells. Annu. Rev. Immunol. 21, 685-711 (2003).

8. Heath, W.R. \& Carbone, F.R. Dendritic cell subsets in primary and secondary $T$ cell responses at body surfaces. Nat. Immunol. 10, 1237-1244 (2009).

9. de Heer, H.J. et al. Essential role of lung plasmacytoid dendritic cells in preventing asthmatic reactions to harmless inhaled antigen. J. Exp. Med. 200, 89-98 (2004).

10. Wollenberg, A. et al. Plasmacytoid dendritic cells: a new cutaneous dendritic cell subset with distinct role in inflammatory skin diseases. J. Invest. Dermatol. 119, 1096-1102 (2002).

11. Villadangos, J.A. \& Young, L. Antigen-presentation properties of plasmacytoid dendritic cells. Immunity 29, 352-361 (2008). 
12. Nudel, I. et al. Dendritic cells in distinct oral mucosal tissues engage different mechanisms to prime CD8 + Tcells. J. Immunol. 186, 891-900 (2011).

13. Aramaki, O., Chalermsarp, N., Otsuki, M., Tagami, J. \& Azuma, M. Differential expression of co-signal molecules and migratory properties in four distinct subsets of migratory dendritic cells from the oral mucosa. Biochem. Biophys. Res. Commun. 413, 407-413 (2011).

14. Mascarell, L. et al. Oral dendritic cells mediate antigen-specific tolerance by stimulating TH1 and regulatory CD4 + Tcells. J. Allergy Clin. Immunol. 122, 603-609 e605 (2008).

15. Song, J.H. et al. CCR7-CCL19/CCL21-regulated dendritic cells are responsible for effectiveness of sublingual vaccination. J. Immunol. 182, 6851-6860 (2009).

16. Arizon, M. et al. Langerhans cells down-regulate inflammation-driven alveolar bone loss. Proc. Natl. Acad. Sci. USA 109, 7043-7048 (2012).

17. Kelsall, B. Recent progress in understanding the phenotype and function of intestinal dendritic cells and macrophages. Mucosal Immunol. 1, 460-469 (2008).

18. Lambrecht, B.N. \& Hammad, H. Lung dendritic cells in respiratory viral infection and asthma: from protection to immunopathology. Annu. Rev. Immunol. 30, 243-270 (2012).

19. Allam, J.P. et al. Distribution of Langerhans cells and mast cells within the human oral mucosa: new application sites of allergens in sublingual immunotherapy?. Allergy 63, 720-727 (2008).

20. Jotwani, R. \& Cutler, C.W. Multiple dendritic cell (DC) subpopulations in human gingiva and association of mature DCs with CD4 + T-cells in situ. J. Dent. Res. 82, 736-741 (2003).

21. Anjana, R., Joseph, L. \& Suresh, R. Immunohistochemical localization of CD1a and S100 in gingival tissues of healthy and chronic periodontitis subjects. Oral Dis. 18, 778-785 (2012).

22. Seguier, S., Bodineau, A., Godeau, G., Pellat, B. \& Brousse, N. Langerin + vs. CD1a + Langerhans cells in human gingival tissue: a comparative and quantitative immunohistochemical study. Arch. Oral Biol. 48, 255-262 (2003).

23. Ito, H. et al. Three-dimensional appearance of Langerhans cells in human gingival epithelium as revealed by confocal laser scanning microscopy. Arch. Oral Biol. 43, 741-744 (1998).

24. Santoro, A. et al. Recruitment of dendritic cells in oral lichen planus. J. Pathol. 205, 426-434 (2005).

25. Kweon, M.N. Sublingual mucosa: A new vaccination route for systemic and mucosal immunity. Cytokine 54, 1-5 (2011).

26. Carbone, F.R., Belz, G.T. \& Heath, W.R. Transfer of antigen between migrating and lymph node-resident DCs in peripheral T-cell tolerance and immunity. Trends Immunol. 25, 655-658 (2004).

27. Summers deLuca, L. \& Gommerman, J.L. Fine-tuning of dendritic cell biology by the TNF superfamily. Nat. Rev. Immunol. 12, 339-351 (2012).

28. Chalermsarp, N. \& Azuma, M. Identification of three distinct subsets of migrating dendritic cells from oral mucosa within the regional lymph nodes. Immunology 127, 558-566 (2009).

29. Desvignes, C., Esteves, F., Etchart, N., Bella, C., Czerkinsky, C. \& Kaiserlian, D. The murine buccal mucosa is an inductive site for priming class I-restricted CD8 + effector T cells in vivo. Clin. Exp. Immunol. 113, 386-393 (1998).

30. Etchart, N. et al. Dendritic cells recruitment and in vivo priming of CD8 + CTL induced by a single topical or transepithelial immunization via the buccal mucosa with measles virus nucleoprotein. J. Immunol. 167, 384-391 (2001).

31. Le Borgne, M. et al. Dendritic cells rapidly recruited into epithelial tissues via CCR6/CCL20 are responsible for CD8 + T cell crosspriming in vivo. Immunity 24, 191-201 (2006).

32. Elnekave, M., Furmanov, K., Nudel, I., Arizon, M., Clausen, B.E. \& Hovav, A.H. Directly transfected langerin + dermal dendritic cells potentiate CD8 + Tcell responses following intradermal plasmid DNA immunization. J. Immunol. 185, 3463-3471 (2010).

33. Dieu, M.C. et al. Selective recruitment of immature and mature dendritic cells by distinct chemokines expressed in different anatomic sites. J. Exp. Med. 188, 373-386 (1998).

34. Saeki, H., Moore, A.M., Brown, M.J. \& Hwang, S.T. Cutting edge: secondary lymphoid-tissue chemokine (SLC) and CC chemokine receptor 7 (CCR7) participate in the emigration pathway of mature dendritic cells from the skin to regional lymph nodes. J. Immunol. 162, 2472-2475 (1999).

35. Otten, K., Dragoo, J., Wang, H.C. \& Klein, J.R. Antigen-induced chemokine activation in mouse buccal epithelium. Biochem. Biophys. Res. Commun. 304, 36-40 (2003).

36. Mascarell, L. et al. Oral macrophage-like cells play a key role in tolerance induction following sublingual immunotherapy of asthmatic mice. Mucosal Immunol. 4, 638-647 (2011).

37. Eriksson, K., Ahlfors, E., George-Chandy, A., Kaiserlian, D. \& Czerkinsky, C. Antigen presentation in the murine oral epithelium. Immunology $\mathbf{8 8}$, 147-152 (1996).

38. Etchart, N., Buckland, R., Liu, M.A., Wild, T.F., Kaiserlian, D. \& Class I-restricted, C.T.L. induction by mucosal immunization with naked DNA encoding measles virus haemagglutinin. J. Gen. Virol. 78 (Pt 7), 1577-1580 (1997).

39. Czerkinsky, C. \& Holmgren, J. Mucosal delivery routes for optimal immunization: targeting immunity to the right tissues. Curr. Top. Microbiol Immunol. 354, 1-18 (2012).

40. Choi, J.H. et al. A single sublingual dose of an adenovirus-based vaccine protects against lethal Ebola challenge in mice and guinea pigs. Mol. Pharm. 9, 156-167 (2012).

41. Appledorn, D.M., Aldhamen, Y.A., Godbehere, S., Seregin, S.S. \& Amalfitano, A. Sublingual administration of an adenovirus serotype 5 (Ad5)-based vaccine confirms Toll-like receptor agonist activity in the oral cavity and elicits improved mucosal and systemic cell-mediated responses against HIV antigens despite preexisting Ad5 immunity. Clin. Vaccine Immunol. 18, 150-160 (2011).

42. Allan, R.S. et al. Migratory dendritic cells transfer antigen to a lymph noderesident dendritic cell population for efficient CTL priming. Immunity 25 , 153-162 (2006).

43. Furmanov, K., Elnekave, M., Lehmann, D., Clausen, B.E., Kotton, D.N. \& Hovav, A.H. The role of skin-derived dendritic cells in CD8 + Tcell priming following immunization with lentivectors. J. Immunol. 184, 4889-4897 (2010).

44. He, Y., Zhang, J., Donahue, C. \& Falo, L.D. Jr. Skin-derived dendritic cells induce potent CD8(+) T cell immunity in recombinant lentivector-mediated genetic immunization. Immunity 24, 643-656 (2006).

45. Hu, B., Tai, A. \& Wang, P. Immunization delivered by lentiviral vectors for cancer and infectious diseases. Immunol. Rev. 239, 45-61 (2011).

46. Lee, H.K. et al. Differential roles of migratory and resident DCs in T cell priming after mucosal or skin HSV-1 infection. J. Exp. Med. 206, 359-370 (2009).

47. Joffre, O.P., Segura, E., Savina, A. \& Amigorena, S. Cross-presentation by dendritic cells. Nat. Rev. Immunol. 12, 557-569 (2012).

48. O'Brien-Simpson, N.M., Pathirana, R.D., Walker, G.D. \& Reynolds, E.C. Porphyromonas gingivalis RgpA-Kgp proteinase-adhesin complexes penetrate gingival tissue and induce proinflammatory cytokines or apoptosis in a concentration-dependent manner. Infect. Immun. 77, 1246-1261 (2009).

49. Allam, J.P. et al. Phl p 5 resorption in human oral mucosa leads to dosedependent and time-dependent allergen binding by oral mucosal Langerhans cells, attenuates their maturation, and enhances their migratory and TGF-beta1 and IL-10-producing properties. J. Allergy Clin. Immunol. 126, 638-645 e631 (2010).

50. Allam, J.P. et al. Toll-like receptor 4 ligation enforces tolerogenic properties of oral mucosal Langerhans cells. J. Allergy Clin. Immunol. 121, 368-374 e361 (2008).

51. Allam, J.P. et al. Characterization of dendritic cells from human oral mucosa: a new Langerhans' cell type with high constitutive FcepsilonRI expression. J. Allergy Clin. Immunol. 112, 141-148 (2003).

52. Hasseus, B., Jontell, M., Bergenholtz, G. \& Dahlgren, U.I. Langerhans cells from human oral epithelium are more effective at stimulating allogeneic T cells in vitro than Langerhans cells from skin. Clin. Exp. Immunol. 136, 483-489 (2004).

53. Hasseus, B., Jontell, M., Bergenholtz, G., Eklund, C. \& Dahlgren, U.I. Langerhans cells from oral epithelium are more effective in stimulating allogeneic t-cells in vitro than Langerhans cells from skin epithelium. J. Dent. Res. 78, 751-758 (1999). 
54. Lutz, M.B. \& Schuler, G. Immature, semi-mature and fully mature dendritic cells: which signals induce tolerance or immunity?. Trends Immunol. $\mathbf{2 3}$, 445-449 (2002).

55. Chino, T. et al. Effects of oral commensal and pathogenic bacteria on human dendritic cells. Oral Microbiol. Immunol. 24, 96-103 (2009).

56. Iliev, I.D., Matteoli, G. \& Rescigno, M. The yin and yang of intestinal epithelial cells in controlling dendritic cell function. J. Exp. Med. 204, 2253-2257 (2007).

57. Dickinson, B.C. et al. Interaction of oral bacteria with gingival epithelial cell multilayers. Mol. Oral Microbiol. 26, 210-220 (2011).

58. Hajishengallis, G. et al. Low-abundance biofilm species orchestrates inflammatory periodontal disease through the commensal microbiota and complement. Cell Host Microbe 10, 497-506 (2011).

59. Jotwani, R. et al. Mature dendritic cells infiltrate the Tcell-rich region of oral mucosa in chronic periodontitis: in situ, in vivo, and in vitro studies. J. Immunol. 167, 4693-4700 (2001).

60. Cirrincione, C., Pimpinelli, N., Orlando, L. \& Romagnoli, P. Lamina propria dendritic cells express activation markers and contact lymphocytes in chronic periodontitis. J. Periodontol. 73, 45-52 (2002).

61. Bodineau, A., Godeau, G., Brousse, N., Pellat, B., Folliguet, M. \& Seguier, S. Langerhans cells express matrix metalloproteinases 9 and 2 and tissue inhibitors of metalloproteinases 1 and 2 in healthy human gingival tissue and in periodontitis. Oral Microbiol. Immunol. 21, 197-200 (2006).

62. Mahanonda, R. et al. Upregulation of co-stimulatory molecule expression and dendritic cell marker (CD83) on B cells in periodontal disease. J. Periodontal Res. 37, 177-183 (2002)

63. Sun, J.B., Flach, C.F., Czerkinsky, C. \& Holmgren, J. B lymphocytes promote expansion of regulatory $\mathrm{T}$ cells in oral tolerance: powerfu induction by antigen coupled to cholera toxin B subunit. J. Immunol. 181, 8278-8287 (2008).

64. Carter, N.A. et al. Mice lacking endogenous IL-10-producing regulatory B cells develop exacerbated disease and present with an increased frequency of Th1/Th17 but a decrease in regulatory T cells. J. Immunol. 186, 5569-5579 (2011).

65. Agrawal, A., Agrawal, S. \& Gupta, S. Dendritic cells in human aging. Exp. Gerontol. 42, 421-426 (2007)

66. Jing, Y., Shaheen, E., Drake, R.R., Chen, N., Gravenstein, S. \& Deng, Y. Aging is associated with a numerical and functional decline in plasmacytoid dendritic cells, whereas myeloid dendritic cells are relatively unaltered in human peripheral blood. Hum. Immunol. 70, 777-784 (2009).

67. Steger, M.M., Maczek, C. \& Grubeck-Loebenstein, B. Morphologically and functionally intact dendritic cells can be derived from the peripheral blood of aged individuals. Clin. Exp. Immunol. 105, 544-550 (1996).

68. Bhushan, M., Cumberbatch, M., Dearman, R.J., Andrew, S.M., Kimber, I. \& Griffiths, C.E. Tumour necrosis factor-alpha-induced migration of human Langerhans cells: the influence of ageing. Br. J. Dermatol. 146, 32-40 (2002).

69. Linton, P.J., Li, S.P., Zhang, Y., Bautista, B., Huynh, Q. \& Trinh, T. Intrinsic vs. environmental influences on T-cell responses in aging. Immunol. Rev. 205, 207-219 (2005)

70. Toapanta, F.R. \& Ross, T.M. Impaired immune responses in the lungs of aged mice following influenza infection. Respir. Res. 10, 112 (2009).

71. Panda, A. et al. Age-associated decrease in TLR function in primary human dendritic cells predicts influenza vaccine response. J. Immunol. 184, 2518-2527 (2010).

72. El Mezayen, R., El Gazzar, M., Myer, R. \& High, K.P. Aging-dependent upregulation of IL-23p19 gene expression in dendritic cells is associated with differential transcription factor binding and histone modifications. Aging Cell 8, 553-565 (2009).

73. Faria, A.M. et al. Aging and immunoglobulin isotype patterns in oral tolerance. Braz. J. Med. Biol. Res. 31, 35-48 (1998).

74. Kato, H. et al. Lack of oral tolerance in aging is due to sequential loss of Peyer's patch cell interactions. Int. Immunol. 15, 145-158 (2003).

75. Della Bella, S. et al. Peripheral blood dendritic cells and monocytes are differently regulated in the elderly. Clin. Immunol. 122, 220-228 (2007).

76. Zavala, W.D. \& Cavicchia, J.C. Deterioration of the Langerhans cell network of the human gingival epithelium with aging. Arch. Oral Biol. 51, 1150-1155 (2006)
77. Cruchley, A.T., Williams, D.M., Farthing, P.M., Speight, P.M., Lesch, C.A. \& Squier, C.A. Langerhans cell density in normal human oral mucosa and skin: relationship to age, smoking and alcohol consumption. J. Oral Pathol. Med. 23, 55-59 (1994).

78. Rittman, B.R., Hill, M.W., Rittman, G.A. \& Mackenzie, I.C. Age-associated changes in Langerhans cells of murine oral epithelium and epidermis. Arch. Oral Biol. 32, 885-889 (1987).

79. Bodineau, A. et al. Do Langerhans cells behave similarly in elderly and younger patients with chronic periodontitis? Arch. Oral Biol. 52, 189-194 (2007).

80. Bodineau, A., Coulomb, B., Tedesco, A.C. \& Seguier, S. Increase of gingival matured dendritic cells number in elderly patients with chronic periodontitis. Arch. Oral Biol. 54, 12-16 (2009).

81. Linden, G.J., McClean, K., Young, I., Evans, A. \& Kee, F. Persistently raised $\mathrm{C}$-reactive protein levels are associated with advanced periodontal disease. J. Clin. Periodontol. 35, 741-747 (2008).

82. Noack, B., Genco, R.J., Trevisan, M., Grossi, S., Zambon, J.J. \& De Nardin, E. Periodontal infections contribute to elevated systemic C-reactive protein level. J. Periodontol. 72, 1221-1227 (2001).

83. Engebretson, S., Chertog, R., Nichols, A., Hey-Hadavi, J., Celenti, R. \& Grbic, J. Plasma levels of tumour necrosis factor-alpha in patients with chronic periodontitis and type 2 diabetes. J. Clin. Periodontol. 34, 18-24 (2007).

84. Kautz-Neu, K. et al. Langerhans cells are negative regulators of the anti-Leishmania response. J. Exp. Med. 208, 885-891 (2011).

85. Shklovskaya, E. et al. Langerhans cells are precommitted to immune tolerance induction. Proc. Natl. Acad. Sci. USA 108, 18049-18054 (2011).

86. Xu, Y.P. et al. Aging affects epidermal Langerhans cell development and function and alters their miRNA gene expression profile. Aging (Albany NY) 4, 742-754 (2012).

87. Toyoda, M. \& Bhawan, J. Ultrastructural evidence for the participation of Langerhans cells in cutaneous photoaging processes: a quantitative comparative study. J. Dermatol. Sci. 14, 87-100 (1997).

88. Warnakulasuriya, S. et al. Oral health risks of tobacco use and effects of cessation. Int. Dent. J. 60, 7-30 (2010).

89. Yanagita, M. et al. Immunomodulation of dendritic cells differentiated in the presence of nicotine with lipopolysaccharide from Porphyromonas gingivalis. Eur. J. Oral Sci. 120, 408-414 (2012).

90. Yanagita, M., Kobayashi, R., Kojima, Y., Mori, K. \& Murakami, S. Nicotine modulates the immunological function of dendritic cells through peroxisome proliferator-activated receptor-gamma upregulation. Cell. Immunol. 274, 26-33 (2012).

91. Danielsen, B., Manji, F., Nagelkerke, N., Fejerskov, O. \& Baelum, V. Effect of cigarette smoking on the transition dynamics in experimental gingivitis. J. Clin. Periodontol. 17, 159-164 (1990).

92. Souto, G.R., Segundo, T.K., Costa, F.O., Aguiar, M.C. \& Mesquita, R.A. Effect of smoking on Langerhans and dendritic cells in patients with chronic gingivitis. J. Periodontol. 82, 619-625 (2011).

93. Daniels, T.E. et al. Reduction of Langerhans cells in smokeless tobaccoassociated oral mucosal lesions. J. Oral Pathol. Med. 21, 100-104 (1992).

94. Carrion, J. et al. Microbial carriage state of peripheral blood dendritic cells (DCs) in chronic periodontitis influences DC differentiation, atherogenic potential. J. Immunol. 189, 3178-3187 (2012).

95. Gemmell, E., Yamazaki, K. \& Seymour, G.J. Destructive periodontitis lesions are determined by the nature of the lymphocytic response. Crit. Rev. Oral Biol. Med. 13, 17-34 (2002).

96. Cochran, D.L. Inflammation and bone loss in periodontal disease. J. Periodontol. 79 (8 Suppl), 1569-1576 (2008).

97. Baker, P.J., Dixon, M., Evans, R.T., Dufour, L., Johnson, E. \& Roopenian, D.C. CD $4(+)$ Tcells and the proinflammatory cytokines gamma interferon and interleukin-6 contribute to alveolar bone loss in mice. Infect. Immun. 67, 2804-2809 (1999).

98. Teng, Y.T. et al. Functional human T-cell immunity and osteoprotegerin ligand control alveolar bone destruction in periodontal infection. J. Clin. Invest. 106, R59-R67 (2000).

99. Daniels, T.E. Human mucosal Langerhans cells: postmortem identification of regional variations in oral mucosa. J. Invest. Dermatol. 82, 21-24 (1984). 
100. Dereka, X.E., Tosios, K.I., Chrysomali, E. \& Angelopoulou, E. Factor XIlla + dendritic cells and S-100 protein + Langerhans' cells in adult periodontitis. J. Periodontal Res. 39, 447-452 (2004).

101. Newcomb, G.M., Seymour, G.J. \& Powell, R.N. Association between plaque accumulation and Langerhans cell numbers in the oral epithelium of attached gingiva. J. Clin. Periodontol. 9, 297-304 (1982).

102. Walsh, L.J., Seymour, G.J. \& Savage, N.W. Oral mucosal Langerhans cells express DR and DQ antigens. J. Dent. Res. 65, 390-393 (1986).

103. Crawford, J.M., Krisko, J.M., Morris, G.A. \& Chambers, D.A. The distribution of Langerhans cells and CD1a antigen in healthy and diseased human gingiva. Reg. Immunol. 2, 91-97 (1989).

104. Cury, P.R., Furuse, C., Rodrigues, A.E., Barbuto, J.A., Araujo, V.C. \& Araujo, N.S. Interstitial and Langerhans' dendritic cells in chronic periodontitis and gingivitis. Braz. Oral Res. 22, 258-263 (2008).

105. Seguier, S., Godeau, G., Leborgne, M., Pivert, G. \& Brousse, N. Quantitative morphological analysis of Langerhans cells in healthy and diseased human gingiva. Arch. Oral Biol. 45, 1073-1081 (2000).

106. Stelin, S., Ramakrishan, H., Talwar, A., Arun, K.V. \& Kumar, T.S. Immunohistological analysis of CD1a langerhans cells and CD57 natural killer cells in healthy and diseased human gingival tissue: a comparative study. J. Indian Soc. Periodontol. 13, 150-154 (2009).

107. Liang, S., Hosur, K.B., Domon, H. \& Hajishengallis, G. Periodontal inflammation and bone loss in aged mice. J. Periodontal Res. 45, 574-578 (2010).

108. van der Velden, U. The onset age of periodontal destruction. J. Clin. Periodontol. 18, 380-383 (1991).

109. Sloberg, K., Jonsson, R. \& Jontell, M. Assessment of Langerhans' cells in oral lichen planus using monoclonal antibodies. J. Oral Pathol. 13, 516-524 (1984).

110. Farthing, P.M., Matear, P. \& Cruchley, A.T. The activation of Langerhans cells in oral lichen planus. J. Oral Pathol. Med. 19, 81-85 (1990).

111. Gustafson, J., Eklund, C., Wallstrom, M., Zellin, G., Magnusson, B. \& Hasseus, B. Langerin-expressing and CD83-expressing cells in oral lichen planus lesions. Acta Odontol. Scand. 65, 156-161 (2007).

112. Walton, L.J., Macey, M.G., Thornhill, M.H. \& Farthing, P.M. Intra-epithelial subpopulations of $\mathrm{T}$ lymphocytes and Langerhans cells in oral lichen planus. J. Oral Pathol. Med. 27, 116-123 (1998).

113. Mukae, S. et al. Detection of fascin and CCR-7 positive mature dendritic cells in oral lichen planus. J. Oral Pathol. Med. 38, 334-342 (2009).

114. Hirota, J., Osaki, T. \& Tatemoto, Y. Immunohistochemical staining of infiltrates in oral lichen planus. Pathol. Res. Pract. 186, 625-632 (1990).
115. Hirota, J. \& Osaki, T. Electron microscopic study on cell-to-cell interactions in oral lichen planus. Pathol. Res. Pract. 188, 1033-1041 (1992).

116. Celenligil, H., Kansu, E., Ruacan, S., Eratalay, K. \& Irkec, M. Characterization of peripheral blood and salivary gland lymphocytes in secondary Sjogren's syndrome. Ann. Dent. 49, 18-22 (1990).

117. Xanthou, G., Tapinos, N.I., Polihronis, M., Nezis, I.P., Margaritis, L.H. \& Moutsopoulos, H.M. CD4 cytotoxic and dendritic cells in the immunopathologic lesion of Sjogren's syndrome. Clin. Exp. Immunol. 118, 154-163 (1999)

118. Gottenberg, J.E. et al. Activation of IFN pathways and plasmacytoid dendritic cell recruitment in target organs of primary Sjogren's syndrome. Proc. Natl. Acad. Sci. USA 103, 2770-2775 (2006).

119. Wildenberg, M.E., van Helden-Meeuwsen, C.G., van de Merwe, J.P., Drexhage, H.A. \& Versnel, M.A. Systemic increase in type I interferon activity in Sjogren's syndrome: a putative role for plasmacytoid dendritic cells. Eur. J. Immunol. 38, 2024-2033 (2008).

120. Siegal, F.P. et al. The nature of the principal type 1 interferon-producing cells in human blood. Science 284, 1835-1837 (1999).

121. Colonna, M., Trinchieri, G. \& Liu, Y.J. Plasmacytoid dendritic cells in immunity. Nat. Immunol. 5, 1219-1226 (2004).

122. Vogelsang, P., Brun, J.G., Oijordsbakken, G., Skarstein, K., Jonsson, R. \& Appel, S. Levels of plasmacytoid dendritic cells and type-2 myeloid dendritic cells are reduced in peripheral blood of patients with primary Sjogren's syndrome. Ann. Rheum. Dis. 69, 1235-1238 (2010).

123. Ozaki, Y. et al. Decrease of blood dendritic cells and increase of tissueinfiltrating dendritic cells are involved in the induction of Sjogren's syndrome but not in the maintenance. Clin. Exp. Immunol. 159, 315-326 (2010).

124. Ozaki, Y. et al. Alteration of peripheral blood dendritic cells in patients with primary Sjogren's syndrome. Arthritis Rheum. 44, 419-431 (2001).

125. Wildenberg, M.E. et al. Increased frequency of CD16 + monocytes and the presence of activated dendritic cells in salivary glands in primary Sjogren syndrome. Ann. Rheum. Dis. 68, 420-426 (2009).

126. Allam, J.P. et al. Tolerogenic T cells, Th1/Th17 cytokines and TLR2/TLR4 expressing dendritic cells predominate the microenvironment within distinct oral mucosal sites. Allergy 66, 532-539 (2011).

127. Lukic, A. et al. Characterization of antigen-presenting cells in human apical periodontitis lesions by flow cytometry and immunocytochemistry. Int. Endod. J. 39, 626-636 (2006). 